\title{
Prognostic Value of Serum Procalcitonin in COVID-19 Patients: A Systematic Review
}

\author{
Sibtain Ahmed ${ }^{1}$, Lena Jafri ${ }^{2}$, Zahra Hoodbhoy ${ }^{3}$, Imran Siddiqui ${ }^{4}$
}

\begin{abstract}
Background: This study is aimed at reviewing the published literature on the prognostic role of serum procalcitonin (PCT) in COVID-19 cases. Data retrieval: We systematically reviewed the literature available on PubMed, MEDLINE, LitCovid NLM, and WHO: to assess the utility of PCT in prognosis of coronavirus disease. Scrutiny for eligible studies comprising articles that have evaluated the prognostic utility of PCT and data compilation was undertaken by two separate investigators. Original articles in human subjects reporting the prognostic role of PCT in adult COVID-19 patients were included. The Quality in Prognosis Studies (QUIPS) tool was utilized to assess the strength of evidence. Results were reported as narrative syntheses.

Results: Out of the total 426 citations, 52 articles passed through screening. The quality of evidence and methodology of included studies was overall acceptable. The total sample size of the studies comprised of 15,296 COVID-19-positive subjects. Majority of the studies were from China, i.e., 40 (77\%). The PCT cut-off utilized was $0.05 \mathrm{ng} / \mathrm{mL}$ by 18 (35\%) studies, followed by $0.5 \mathrm{ng} / \mathrm{mL}$ by 9 (17.5\%). Eighty five percent $(n=44)$ studies reported statistically significant association ( $p$ value $<0.05$ ) between PCT and severity.

Conclusion: Procalcitonin appears as a promising prognostic biomarker of COVID-19 progression in conjunction with the clinical context.

Keywords: COVID-19, Procalcitonin, Prognostic biomarker, Systematic review.

Indian Journal of Critical Care Medicine (2021): 10.5005/jp-journals-10071-23706
\end{abstract}

\section{INTRODUCTION}

The coronavirus disease 2019 (COVID-19) emerged as an unanticipated emergency crisis at the beginning of year 2020 with devastating medical, social, and financial implications globally. ${ }^{1}$ From a clinical perspective, the unknown complications, lack of availability of prophylactic and reliable therapeutic regimens, and the intricacy of the severe acute respiratory syndrome coronavirus 2 (SARS-CoV-2) infection, accompanied by a rapidly evolving clinical course, puzzled the medical community worldwide. ${ }^{2}$

The clinical scientists extensively and urgently studied reliable biochemical markers related to COVID-19 disease severity aimed at high-risk stratifications and optimal resource allocation, in the already overwhelmed medical infrastructure. ${ }^{3}$ The biomarkers that were particularly explored in this context included procalcitonin (PCT), C-reactive protein (CRP), ferritin (Fer), D-dimer, interleukin-6 (IL-6), and lactate dehydrogenase (LDH). ${ }^{3}$

Preliminary studies have described pathogenetic mechanisms triggered by COVID-19 including a plethora of inflammatory processes, cytokine storms, and the stimulation of coagulation pathways; ultimately, a picture of systematic inflammation ensues with systemic vasculitis and often fatal complications. ${ }^{4}$ Due to the characteristic nature of $\mathrm{PCT}$ in bacterial vs. viral infections, this biomarker may have a role in prognosis of COVID-19. ${ }^{5}$

Procalcitonin is a glycoprotein calcitonin pro-hormone released by the thyroid parafollicular cells. In case of a microbial infection, PCT levels are significantly raised as it is released by all parenchymal tissue under the influence of endotoxins and pro-inflammatory cytokines. ${ }^{6}$ Thus, in physiological state serum PCT is recorded significantly below $0.05 \mathrm{ng} / \mathrm{mL}$. Furthermore, keeping in view the timelines for risk stratification, PCT follows a swift course with its inclining levels detected 2-6 hours after the stimulus. ${ }^{6}$ However,

\author{
1,2,4 Department of Pathology and Laboratory Medicine, The Aga Khan \\ University, Karachi, Pakistan \\ ${ }^{3}$ Department of Pediatrics and Child Health, The Aga Khan University, \\ Karachi, Pakistan \\ Corresponding Author: Lena Jafri, Department of Pathology and \\ Laboratory Medicine, The Aga Khan University, Karachi, Pakistan, \\ Phone: +92-213-4861927, e-mail: lena.jafri@aku.edu \\ How to cite this article: Ahmed S, Jafri L, Hoodbhoy Z, Siddiqui I. \\ Prognostic Value of Serum Procalcitonin in COVID-19 Patients: A \\ Systematic Review. Indian J Crit Care Med 2021;25(1):77-84. \\ Source of support: Nil \\ Conflict of interest: None
}

highly regarded and utilized as a biomarker of bacterial infection, contrasting opinion exists on the efficacy of PCT as a prognostic tool for COVID-19. ${ }^{7,8}$ Moreover, cytokines released in COVID-19, particularly interferon (INF)- $\gamma$, have a negative effect on PCT levels, adding to the strength of this prognostic tool. ${ }^{9}$ Early studies in the wake of the pandemic have shown higher levels of PCT in severe COVID-19 cases. $^{10}$ Lippi et al. have reported that the PCT levels are expected to quintuple in severe cases. ${ }^{11}$ Various other authors have also supported the view that any considerable increase from baseline PCT levels reflects the onset of a critical phase of the viral infection. $^{12}$

Given these unique characteristics, reliable kinetics, and the potential association of declining levels with resolution of infection, PCT has emerged as a promising prognostic biomarker in COVID-19. ${ }^{11}$ This paper is aimed at an extensive evaluation of the published literature on the prognostic role of serum PCT in COVID-19 cases.

(c) The Author(s). 2021 Open Access This article is distributed under the terms of the Creative Commons Attribution 4.0 International License (https://creativecommons. org/licenses/by-nc/4.0/), which permits unrestricted use, distribution, and non-commercial reproduction in any medium, provided you give appropriate credit to the original author(s) and the source, provide a link to the Creative Commons license, and indicate if changes were made. The Creative Commons Public Domain Dedication waiver (http://creativecommons.org/publicdomain/zero/1.0/) applies to the data made available in this article, unless otherwise stated. 


\section{Data Retrieval}

The team of investigators performed a systematic literature review based on Medline (PubMed interface), LitCovid NLM, and WHO: Global literature on coronavirus disease from the advent of COVID19 in December 2019 till June 15, 2020. The strategy adopted was in line with the Preferred Reporting Items for Systematic Reviews and Meta-Analyses (PRISMA) guidelines. ${ }^{13}$

Keywords and medical subject heading terms searched included Coronavirus OR "corona virus" OR coronavirinae OR coronaviridae OR betacoronavirus OR covid19 OR "covid 19" OR nCoV OR "CoV 2" OR CoV2 OR sarscov2 OR 2019nCoV OR "novel CoV" OR "wuhan virus") OR ((wuhan OR hubei OR huanan) AND ("severe acute respiratory" OR pneumonia) AND (outbreak)) OR "Coronavirus" OR "Coronavirus Infections" OR "COVID-19" OR "severe acute respiratory syndrome coronavirus 2" OR "Betacoronavirus"AND (Procalcitonin OR PCT OR Calcitonin Precursor Polyprotein OR Calcitonin-1 OR Calcitonin 1 OR Calcitonin Related Polypeptide Alpha OR ProCalcitonin) without language restrictions.

Nonhuman and biological model studies were not included. Moreover, two separate investigators (SA and LJ) reviewed the titles and abstracts of all articles identified for inclusion in the final analysis; alongside, the references of the scrutinized articles and the PubMed-related article feature were also explored for any additional publications of potential interest. The inclusion criteria were structured upon the following conditions: (1) study participants (adults with confirmed COVID-19); (2) single or serial measurements of serum PCT documented; (3) assessment of prognostic performance of PCT; (4) at least one outcome measure documented, i.e., severe infection requiring mechanical ventilation, needing admission to intensive care unit, and mortality; and (5) study design: cross sectional, cohort, casecontrol, and case series.

Studies comprising reviews, meta-analysis, letters to the editor, surveys, commentary, perspectives, opinion papers, hypothesis, viewpoints, animal studies, drug discovery, drug trials, basic sciences/nonclinical studies, studies done in pediatric population, article full text in language other than English, and abstracts only were omitted. Full-text article versions of the abstracts included in the final study analysis were further appraised by two authors. The agreement and concordance between independent evaluations was statistically sought with $\kappa$ statistic for the interrater reliability. ${ }^{14}$

The quality of evidence gathered and likely risk of bias was evaluated according to the quality in prognosis studies (QUIPS) tool. ${ }^{15}$ Scores were calculated as "low," "moderate," or "high" for 30 variables under six domains, namely study population selection, attrition rate, prognostic biomarker analysis, outcome assessment, potential confounders evaluation, statistical exploration, and delineation. High quality was defined as attainment of low or moderate risk of bias for most domains and vice versa. A third reviewer (IS) was involved to resolve the disagreement in opinions through mutual consensus.

The two reviewers autonomously compiled the data using a predesigned pro forma enlisting the region of study publication, number of study participants, time period of recruitment, PCT cut-offs utilized, and correlation of PCT with severity by the $p$ value and descriptive results as reported by the different studies included in the final analysis.

\section{Results}

The databases searched revealed a total of 426 studies. Moreover, 26 duplicate studies were excluded. Based on the stringent inclusion criteria as depicted in Flowchart 1, 52 articles were included in the final analysis based on autonomous evaluation by two investigators with an excellent agreement of $\kappa$ statistic $=0.90$. The accumulated sample size of the studies comprised of 15,296 cases.

Table 1 presents a comprehensive overview of the articles included in this systematic review published from January to June 2020. Different PCT cut-offs were utilized ranging from 0.05 , 0.1 and $0.5 \mathrm{ng} / \mathrm{mL}$, by $35 \%(n=18), 9 \%(n=5)$, and $17.5 \%(n=9)$ studies, respectively, whereas, cut-off used was not reported by $27 \%(n=14)$ studies.

Seventy-seven percent $(n=40)$ were reported from China as shown in Figure 1. Total $44(85 \%)$ studies reported statistically significant association ( $p$ value $<0.05$ ) between PCT and severity

Flowchart 1: Search strategy

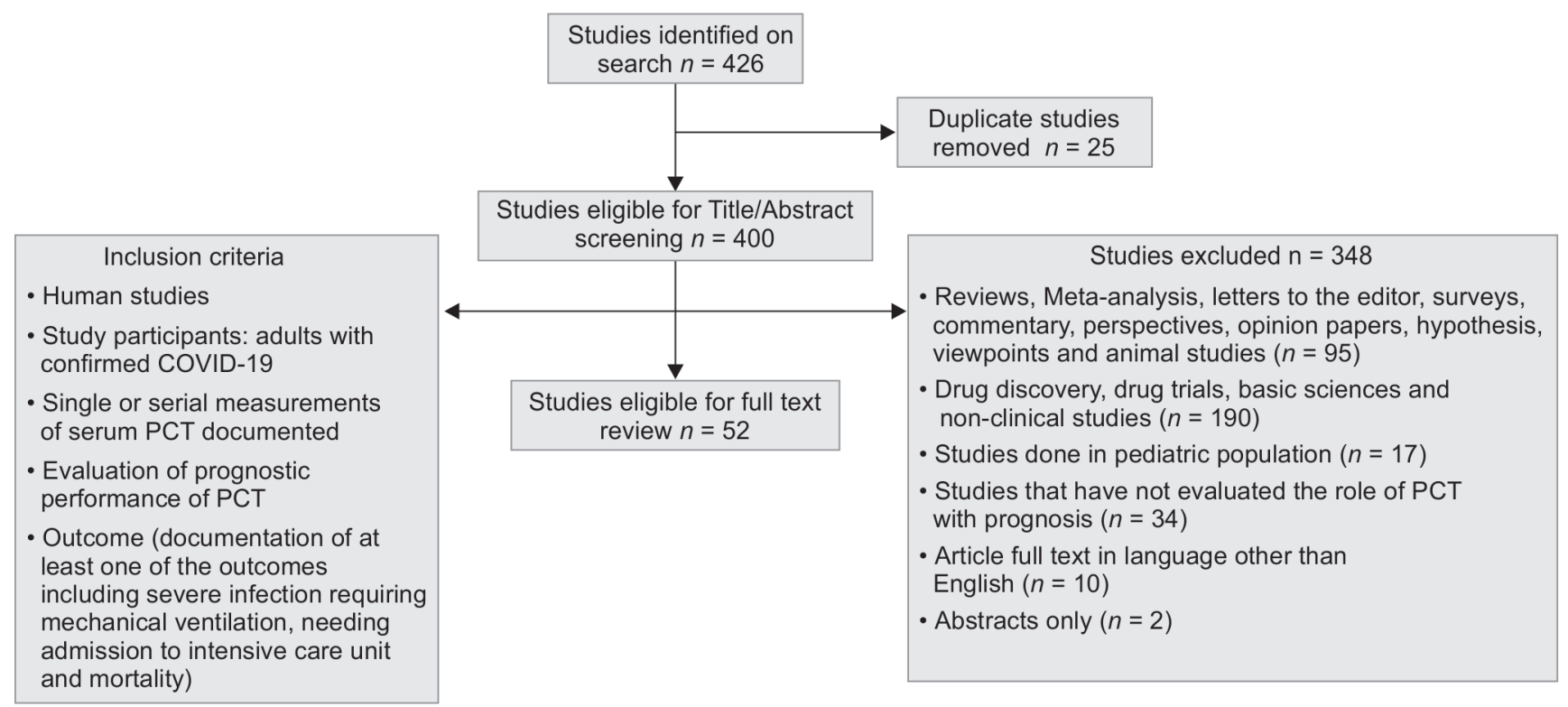


Table 1: Summary of studies evaluated and enlisting correlation of PCT with severity

\begin{tabular}{|c|c|c|c|c|c|c|c|c|}
\hline Author & Region & $\begin{array}{l}\text { Date of } \\
\text { recruitment }\end{array}$ & $\begin{array}{l}\text { Year of } \\
\text { recruitment } \\
\text { initiation }\end{array}$ & Study design & $\begin{array}{l}\text { Sample size } \\
\text { (male:female) }\end{array}$ & $\begin{array}{l}\text { Age in years, } \\
\text { Median } \\
\text { (range) or } \\
\text { Mean } \pm S D\end{array}$ & $\begin{array}{l}\text { Cut-off used } \\
\text { for elevated } \\
P C T(\mathrm{ng} / \mathrm{mL})\end{array}$ & $\begin{array}{l}\text { PCT correlation with } \\
\text { severity ( } p \text { value = } \\
\text { correlation } \\
\text { coefficient) }\end{array}$ \\
\hline $\begin{array}{l}\text { Bhandari } \\
\text { et al. }\end{array}$ & India & March to May & 2020 & $\begin{array}{l}\text { Prospective } \\
\text { cohort }\end{array}$ & $21(14: 7)$ & $\begin{array}{l}43.5 \\
(2.0-85.0)\end{array}$ & $\mathrm{N} / \mathrm{R}$ & $\begin{array}{l}\mathrm{PCT} \text { elevated in all } \\
\text { severe cases }\end{array}$ \\
\hline $\begin{array}{l}\text { Cecconi } \\
\text { et al. }{ }^{16}\end{array}$ & Italy & $\begin{array}{l}\text { February to } \\
\text { March }\end{array}$ & 2020 & $\begin{array}{l}\text { Retrospective } \\
\text { cohort }\end{array}$ & $239(169: 70)$ & $63.9 \pm 14.0$ & $>0.5$ & $p<0.001$ \\
\hline Cen et al. ${ }^{17}$ & China & February & 2020 & $\begin{array}{l}\text { Prospective } \\
\text { cohort }\end{array}$ & $\begin{array}{l}1007 \\
(493: 514)\end{array}$ & $61(49-68)$ & $>0.5$ & $p<0.044$ \\
\hline Chen et al. ${ }^{18}$ & China & March & 2020 & $\begin{array}{l}\text { Retrospective } \\
\text { cohort }\end{array}$ & $548(313: 235)$ & $56.0 \pm 14.5$ & $>0.5$ & $p<0.001$ \\
\hline Chen et al. ${ }^{19}$ & China & $\begin{array}{l}\text { December to } \\
\text { January }\end{array}$ & 2019 & $\begin{array}{l}\text { Retrospective } \\
\text { cohort }\end{array}$ & 1590 (N/R) & $69(51-86)$ & $>0.5$ & $p<0.001$ \\
\hline Chen et al. ${ }^{20}$ & China & $\begin{array}{l}\text { January to } \\
\text { February }\end{array}$ & 2020 & $\begin{array}{l}\text { Retrospective } \\
\text { cohort }\end{array}$ & $203(108: 95)$ & $54(20-91)$ & $>1.0$ & $p<0.04$ \\
\hline Chen et al. ${ }^{21}$ & China & January & 2020 & $\begin{array}{l}\text { Retrospective } \\
\text { cohort }\end{array}$ & 78 (39:39) & $45(15-79)$ & $>0.5$ & $p<0.001$ \\
\hline Duan et al. ${ }^{22}$ & China & $\begin{array}{l}\text { January to } \\
\text { February }\end{array}$ & 2020 & $\begin{array}{l}\text { Retrospective } \\
\text { cohort }\end{array}$ & $397(233: 164)$ & $51.0 \pm 15$ & $>0.04$ & $p<0.01$ \\
\hline Gavin et al. ${ }^{23}$ & USA & March & 2020 & $\begin{array}{l}\text { Retrospective } \\
\text { cohort }\end{array}$ & $140(72: 68)$ & $60(48-72)$ & $>0.24$ & $p<0.00004$ \\
\hline $\begin{array}{l}\text { Gregoriano et } \\
\text { al. }^{24}\end{array}$ & Switzerland & $\begin{array}{l}\text { February to } \\
\text { April }\end{array}$ & 2020 & $\begin{array}{l}\text { Retrospective } \\
\text { cohort }\end{array}$ & $99(62: 37)$ & $67(56-76)$ & $>0.05$ & $p<0.002$ \\
\hline Guo, et al. ${ }^{25}$ & China & $\begin{array}{l}\text { January to } \\
\text { February }\end{array}$ & 2020 & $\begin{array}{l}\text { Retrospective } \\
\text { case series }\end{array}$ & $187(91: 96)$ & $58.50 \pm 14.66$ & $>0.05$ & $p<0.001$ \\
\hline Hong, et al. ${ }^{26}$ & China & $\begin{array}{l}\text { January to } \\
\text { February }\end{array}$ & 2020 & $\begin{array}{l}\text { Retrospective } \\
\text { cohort }\end{array}$ & $75(41: 34)$ & $46.37 \pm 13.34$ & $N / R$ & $p<0.004$ \\
\hline Hou et al. ${ }^{27}$ & China & $\begin{array}{l}\text { January to } \\
\text { February }\end{array}$ & 2020 & $\begin{array}{l}\text { Prospective } \\
\text { cohort }\end{array}$ & 389 (200:189) & $61 \cdot 3 \pm 13 \cdot 8$ & $\mathrm{~N} / \mathrm{R}$ & $p<0.0001$ \\
\hline Hu et al. ${ }^{28}$ & China & $\begin{array}{l}\text { January to } \\
\text { March }\end{array}$ & 2020 & $\begin{array}{l}\text { Retrospective } \\
\text { cohort }\end{array}$ & 95 (39:56) & $57.6 \pm 14.7$ & $>0.05$ & $p<0.05$ \\
\hline Ke et al. ${ }^{10}$ & China & January & 2020 & Case series & $2(1: 1)$ & 79 and 40 & $>0.05$ & $\begin{array}{l}\text { PCT was elevated } \\
\text { in all cases }\end{array}$ \\
\hline Li et al. ${ }^{29}$ & China & $\begin{array}{l}\text { January to } \\
\text { February }\end{array}$ & 2020 & $\begin{array}{l}\text { Retrospective } \\
\text { cohort }\end{array}$ & $132(75: 57)$ & $62(33-89)$ & $>0.05$ & $\begin{array}{l}\text { PCT had no sig- } \\
\text { nificant changes in } \\
\text { association disease } \\
\text { severity }\end{array}$ \\
\hline Li et al. ${ }^{30}$ & China & $\begin{array}{l}\text { January to } \\
\text { February }\end{array}$ & 2020 & $\begin{array}{l}\text { Retrospective } \\
\text { cohort }\end{array}$ & $225(120: 105)$ & $50 \pm 14$ & $>0.5$ & $\begin{array}{l}\text { PCT was elevated } \\
\text { in } 10.67 \% \text { of } \\
\text { patients }\end{array}$ \\
\hline Li et al. ${ }^{12}$ & China & $\begin{array}{l}\text { January to } \\
\text { February }\end{array}$ & 2020 & $\begin{array}{l}\text { Retrospective } \\
\text { cohort }\end{array}$ & $25(10: 15)$ & $73(55-100)$ & $>0.1$ & $\begin{array}{l}\text { PCT was elevated } \\
\text { in } 90.5 \% \text { of } \\
\text { patients }\end{array}$ \\
\hline Lima et al. ${ }^{31}$ & USA & March to April & 2020 & $\begin{array}{l}\text { Retrospective } \\
\text { cohort }\end{array}$ & $5(4: 1)$ & $45-68$ & $>0.5$ & $\begin{array}{l}\text { PCT was elevated } \\
\text { in two cases }\end{array}$ \\
\hline Liu et al. ${ }^{32}$ & China & $\begin{array}{l}\text { January to } \\
\text { March }\end{array}$ & 2020 & $\begin{array}{l}\text { Retrospective } \\
\text { observational }\end{array}$ & $141(49: 91)$ & $\begin{array}{l}65.5(54.3- \\
73.0)\end{array}$ & $>0.07$ & $p=0.025$ \\
\hline Liu et al. ${ }^{33}$ & China & February & 2020 & $\begin{array}{l}\text { Retrospective } \\
\text { cohort }\end{array}$ & $107(52: 55)$ & $68(61-76)$ & $>0.1$ & $p=0.031$ \\
\hline Luo et al. ${ }^{34}$ & China & $\begin{array}{l}\text { January to } \\
\text { February }\end{array}$ & 2020 & $\begin{array}{l}\text { Retrospective } \\
\text { cohort }\end{array}$ & $298(150: 148)$ & $57(40-69)$ & $N / R$ & $p<0.001$ \\
\hline Ma et al. ${ }^{35}$ & China & $\begin{array}{l}\text { January to } \\
\text { March }\end{array}$ & 2020 & $\begin{array}{l}\text { Retrospective } \\
\text { cohort }\end{array}$ & $37(20: 17)$ & $62(59-70)$ & $>0.1$ & $p<0.001$ \\
\hline McRae et al. ${ }^{36}$ & USA & April & 2020 & $\begin{array}{l}\text { Retrospective } \\
\text { cohort }\end{array}$ & $160(82: 78)$ & $63 \pm 13$ & $>0.05$ & $p<0.001$ \\
\hline
\end{tabular}


Contd...

\begin{tabular}{|c|c|c|c|c|c|c|c|c|}
\hline Author & Region & $\begin{array}{l}\text { Date of } \\
\text { recruitment }\end{array}$ & $\begin{array}{l}\text { Year of } \\
\text { recruitment } \\
\text { initiation }\end{array}$ & Study design & $\begin{array}{l}\text { Sample size } \\
\text { (male:female) }\end{array}$ & $\begin{array}{l}\text { Age in years, } \\
\text { Median } \\
\text { (range) or } \\
\text { Mean } \pm S D\end{array}$ & $\begin{array}{l}\text { Cut-off used } \\
\text { for elevated } \\
P C T(n g / m L)\end{array}$ & $\begin{array}{l}\text { PCT correlation with } \\
\text { severity ( } p \text { value = } \\
\text { correlation } \\
\text { coefficient) }\end{array}$ \\
\hline Ni et al. ${ }^{37}$ & China & February & 2020 & $\begin{array}{l}\text { Prospective } \\
\text { cohort }\end{array}$ & $27(14: 13)$ & $60(33-83)$ & $>0.05$ & $p<0.01$ \\
\hline $\begin{array}{l}\text { Ortiz-Brizuela } \\
\text { et al. }{ }^{38}\end{array}$ & Mexico & $\begin{array}{l}\text { February to } \\
\text { April }\end{array}$ & $2020 a$ & $\begin{array}{l}\text { Prospective } \\
\text { cohort }\end{array}$ & 309 (183:126) & $43(33-54)$ & $>0.05$ & $p<0.001$ \\
\hline Pan et al..$^{39}$ & China & $\begin{array}{l}\text { January to } \\
\text { March }\end{array}$ & 2020 & $\begin{array}{l}\text { Case-control } \\
\text { study }\end{array}$ & $124(85: 39)$ & $68(61-75)$ & $>0.05$ & $p<0.001$ \\
\hline $\begin{array}{l}\text { Price-Hay- } \\
\text { wood et al. }{ }^{40}\end{array}$ & $\begin{array}{l}\text { United } \\
\text { States }\end{array}$ & March to April & 2020 & $\begin{array}{l}\text { Retrospective } \\
\text { cohort }\end{array}$ & $\begin{array}{l}3,491 \\
(2097: 1394)\end{array}$ & $\begin{array}{l}55.5 \pm 18.5 \\
53.6 \pm 16.1\end{array}$ & $>0.25$ & $\begin{array}{l}\text { Elevated PCT } \\
\text { associated with in- } \\
\text { hospital mortality } \\
\text { HR } 1.40 \text { (1.06-1.84) }\end{array}$ \\
\hline $\begin{array}{l}\text { Rastrelli et } \\
\text { al. }{ }^{41}\end{array}$ & Italy & $\mathrm{N} / \mathrm{R}$ & 2020 & Case series & 31 (All males) & $63(55-66.5)$ & $>0.9$ & $p<0.001$ \\
\hline Rath et al. ${ }^{42}$ & Germany & $\begin{array}{l}\text { February to } \\
\text { March }\end{array}$ & 2020 & $\begin{array}{l}\text { Prospective } \\
\text { cohort }\end{array}$ & $123(77: 46)$ & $68 \pm 15$ & $N / R$ & $p<0.002$ \\
\hline Sattar et al. ${ }^{43}$ & USA & $N / R$ & 2020 & Case report & 1 (male) & 67 & $N / R$ & $\begin{array}{l}\text { PCT levels } \\
\text { increased with dis- } \\
\text { ease progression }\end{array}$ \\
\hline Shao et al. ${ }^{44}$ & China & $\begin{array}{l}\text { January to } \\
\text { March }\end{array}$ & 2020 & $\begin{array}{l}\text { Retrospective } \\
\text { cohort }\end{array}$ & $155(62: 93)$ & $48(7-96)$ & $>0.05$ & $p=0.032$ \\
\hline Sun et al. ${ }^{45}$ & China & $\begin{array}{l}\text { January to } \\
\text { February }\end{array}$ & 2020 & $\begin{array}{l}\text { Retrospective } \\
\text { cohort }\end{array}$ & $84(47: 37)$ & $64(21-95)$ & $N / R$ & $p<0.001$ \\
\hline Tian et al. ${ }^{46}$ & China & $\begin{array}{l}\text { January to } \\
\text { March }\end{array}$ & 2020 & $\begin{array}{l}\text { Retrospective } \\
\text { cohort }\end{array}$ & $232(119: 113)$ & $64(58-69)$ & $N / R$ & $p<0.001$ \\
\hline Wan et al. ${ }^{47}$ & China & $\begin{array}{l}\text { January to } \\
\text { February }\end{array}$ & 2020 & Case series & $135(72: 63)$ & $47(36-55)$ & $N / R$ & $p<0.05$ \\
\hline Wang et al. ${ }^{48}$ & China & $\begin{array}{l}\text { January to } \\
\text { February }\end{array}$ & 2020 & $\begin{array}{l}\text { Prospective } \\
\text { cohort }\end{array}$ & $85(45: 40)$ & $59 \pm 15.3$ & $N / R$ & $p<0.011$ \\
\hline Wang et al. ${ }^{49}$ & China & $\begin{array}{l}\text { January to } \\
\text { March }\end{array}$ & 2020 & $\begin{array}{l}\text { Retrospective } \\
\text { cohort }\end{array}$ & $108(72: 36)$ & $\begin{array}{l}70.9 \pm 10.6 \\
71.1 \pm 10.1\end{array}$ & $\mathrm{~N} / \mathrm{R}$ & $p<0.001$ \\
\hline Wang et al. ${ }^{50}$ & China & $\begin{array}{l}\text { January to } \\
\text { February }\end{array}$ & 2020 & $\begin{array}{l}\text { Retrospective } \\
\text { cohort }\end{array}$ & $28(21: 7)$ & $68.6 \pm 9.0$ & $N / R$ & $p=0.0006$ \\
\hline Wu et al. ${ }^{51}$ & China & $\begin{array}{l}\text { January to } \\
\text { February }\end{array}$ & 2020 & $\begin{array}{l}\text { Retrospective } \\
\text { cohort }\end{array}$ & $270(139: 131)$ & $62(150-69)$ & $>0.05$ & $p<0.05$ \\
\hline Yan et al. ${ }^{52}$ & China & $\begin{array}{l}\text { January to } \\
\text { February }\end{array}$ & 2020 & $\begin{array}{l}\text { Retrospective } \\
\text { observational }\end{array}$ & $193(114: 79)$ & $64(49-73)$ & $>0.05$ & $p<0.001$ \\
\hline Yang et al. ${ }^{53}$ & China & $\begin{array}{l}\text { January to } \\
\text { February }\end{array}$ & 2020 & $\begin{array}{l}\text { Retrospective } \\
\text { cohort }\end{array}$ & $114(56: 58)$ & $46.05 \pm 15.15$ & $>5$ & $p<0.014$ \\
\hline Yang et al. ${ }^{54}$ & China & $\begin{array}{l}\text { January to } \\
\text { April }\end{array}$ & 2020 & $\begin{array}{l}\text { Retrospective } \\
\text { cohort }\end{array}$ & $52(28: 24)$ & $63(34-98)$ & $N / R$ & $p<0.05$ \\
\hline Yang et al. ${ }^{55}$ & China & $\begin{array}{l}\text { January to } \\
\text { February }\end{array}$ & 2020 & $\begin{array}{l}\text { Retrospective } \\
\text { case series }\end{array}$ & $136(66: 70)$ & $56(44-64)$ & $>0.05$ & $p<0.001$ \\
\hline Ye et al. ${ }^{56}$ & China & $\begin{array}{l}\text { January to } \\
\text { March }\end{array}$ & 2020 & $\begin{array}{l}\text { Retrospective } \\
\text { cohort }\end{array}$ & $349(173: 176)$ & $62(21: 69)$ & $N / R$ & $p<0.001$ \\
\hline Yu et al. ${ }^{57}$ & China & $\begin{array}{l}\text { January to } \\
\text { February }\end{array}$ & 2020 & $\begin{array}{l}\text { Retrospective } \\
\text { cohort }\end{array}$ & $\begin{array}{l}1663 \\
(736: 728)\end{array}$ & $64(51-71)$ & $>0.05$ & $p<0.001$ \\
\hline Yuan et al. ${ }^{58}$ & China & $\begin{array}{l}\text { February to } \\
\text { March }\end{array}$ & 2020 & $\begin{array}{l}\text { Retrospective } \\
\text { cohort }\end{array}$ & $117(56: 61)$ & $66(29-92)$ & $>0.5$ & $p<0.01$ \\
\hline $\begin{array}{l}\text { Zaninotto } \\
\text { et al. } .^{59}\end{array}$ & Italy & $\begin{array}{l}\text { January to } \\
\text { March }\end{array}$ & 2020 & $\begin{array}{l}\text { Prospective } \\
\text { cohort }\end{array}$ & $75(56: 19)$ & $67(56-76)$ & $>0.5$ & $\begin{array}{l}\text { PCT was elevated } \\
\text { in } 2 \text { severe cases }\end{array}$ \\
\hline Zeng et al. ${ }^{60}$ & China & $\begin{array}{l}\text { January to } \\
\text { March }\end{array}$ & 2020 & $\begin{array}{l}\text { Retrospective } \\
\text { cohort }\end{array}$ & $461(239: 222)$ & $45(34.5-57)$ & $>0.05$ & $p<0.001$ \\
\hline
\end{tabular}


Contd...

\begin{tabular}{|c|c|c|c|c|c|c|c|c|}
\hline Author & Region & $\begin{array}{l}\text { Date of } \\
\text { recruitment }\end{array}$ & $\begin{array}{l}\text { Year of } \\
\text { recruitment } \\
\text { initiation }\end{array}$ & Study design & $\begin{array}{l}\text { Sample size } \\
\text { (male:female) }\end{array}$ & $\begin{array}{l}\text { Age in years, } \\
\text { Median } \\
\text { (range) or } \\
\text { Mean } \pm S D\end{array}$ & $\begin{array}{l}\text { Cut-off used } \\
\text { for elevated } \\
\text { PCT }(\mathrm{ng} / \mathrm{mL})\end{array}$ & $\begin{array}{l}\text { PCT correlation with } \\
\text { severity ( } p \text { value }= \\
\text { correlation } \\
\text { coefficient) }\end{array}$ \\
\hline Zhang et al. ${ }^{61}$ & China & $\begin{array}{l}\text { January to } \\
\text { February }\end{array}$ & 2020 & $\begin{array}{l}\text { Retrospective } \\
\text { cohort }\end{array}$ & $84(50: 34)$ & $49(24-80)$ & $>0.05$ & $p<0.003$ \\
\hline Zhang et al. ${ }^{62}$ & China & $\begin{array}{l}\text { January to } \\
\text { February }\end{array}$ & 2020 & $\begin{array}{l}\text { Retrospective } \\
\text { case series }\end{array}$ & $221(108: 113)$ & $55(39-66.5)$ & $>0.05$ & $p<0.001$ \\
\hline Zhang et al. ${ }^{63}$ & China & $\begin{array}{l}\text { December to } \\
\text { February }\end{array}$ & 2019 & $\begin{array}{l}\text { Retrospective } \\
\text { cohort }\end{array}$ & $289(155: 134)$ & $57(22-88)$ & $>0.1$ & $p<0.004$ \\
\hline Zhang et al. ${ }^{64}$ & China & $\begin{array}{l}\text { January to } \\
\text { February }\end{array}$ & 2020 & $\begin{array}{l}\text { Retrospective } \\
\text { cohort }\end{array}$ & $140(71: 69)$ & $57(25-87)$ & $>0.1$ & $p<0.001$ \\
\hline
\end{tabular}

*Studies in alphabetic order; *N/R: not recorded; $\mathrm{PCT}$, procalcitonin

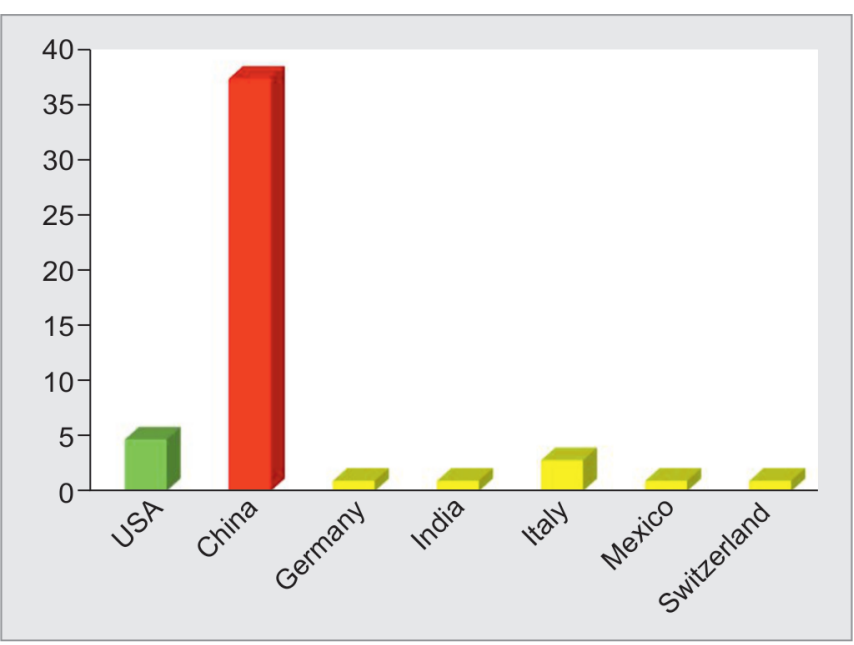

Fig. 1: Countries of origin of studies

based on $p$ values or hazard's ratio. Seven (14\%) studies did not present a statistical analysis however reported elevation of PCT in severe case or mortality. Whereas, only one study did not report any significant elevation in PCT with disease progression.

Figure 2 shows the quality of evidence according to the QUIPS tool. Low to moderate risk of bias was noted for most domains whereas high risk in one or more domains was evident in only eight studies (15\%). For the probable effects of the confounding variable, no adjustments in the analysis were undertaken. The statistical analysis realm had a moderate to high risk of bias in five (9\%) articles. Eight (15\%) studies did not report use of any statistical analysis to assess PCT's association with prognosis and only narrative results were presented while moderate to high risk was noted for the statistical analysis in five (9\%) studies.

Three studies evaluated the receiver operating characteristic (ROC) curve for PCT and reported optimal cut-off and the area under the curve (AUC) for the prediction of severity. ${ }^{22,24,32}$ The optimal cut-offs reported alongside the AUC were $0.04 \mathrm{ng} / \mathrm{mL}$ (AUC 0.74 [95\% Cl: $0.69-0.78]$ ), $0.11 \mathrm{ng} / \mathrm{mL}$ (AUC: 0.80 [95\% Cl: $0.71-0.90]$ ), and $0.07 \mathrm{ng} / \mathrm{mL}$ (AUC: 0.74 ), respectively.

\section{Discussion}

With the global medical crisis amidst the COVID-19 pandemic, the role of laboratory evaluation and early prediction of the severity of a patients' condition was markedly highlighted. Since its inception on the diagnostic platform in 1993, a significant and reliable linkage

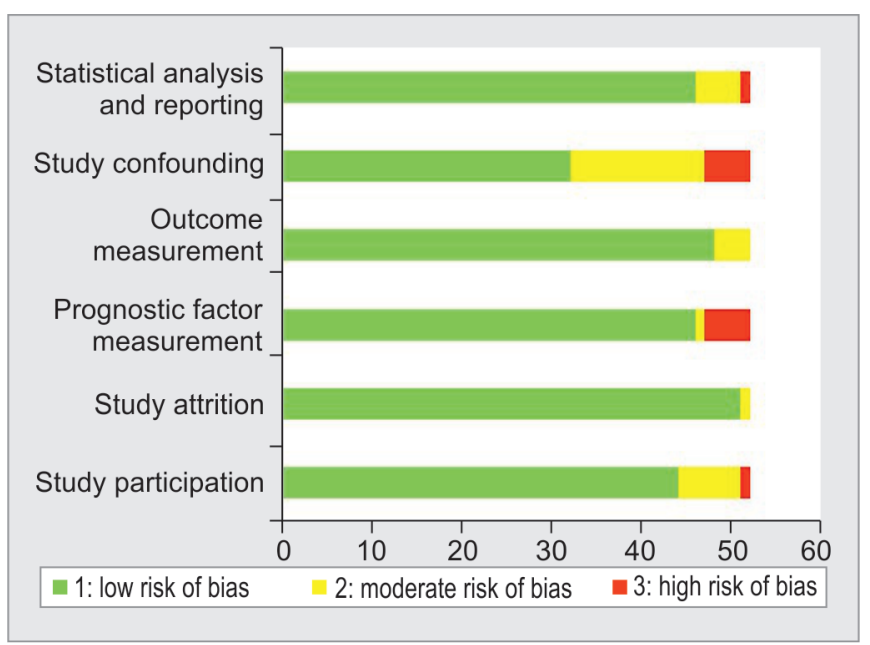

Fig. 2: Assessment of risk of bias using QUIPS tool

between serum PCT level for severity prediction of infectious etiologies has been widely reported. ${ }^{65}$ In context of COVID-19, the extrathyroidal secretion of PCT is thought to be massively intensified during infectious insults and, furthermore, actively precipitated by the inflammatory cytokines. ${ }^{3}$

To further substantiate this association, our results revealed 51 studies reported either a significant association between PCT and severity or markedly elevated PCT levels in the severe patients group making it a reliable prognostic biomarker. However, Li et al. reported no significant association between PCT and prognosis in COVID-19 cases, which could be due to a small proportion of critically ill $(n=5)$ and expired $(n=11)$ cases and the findings could vary if a larger cohort is evaluated. ${ }^{29} \mathrm{~A}$ handful of studies presented clinical and outcome profiles of COVID-19 cases in their respective setups, accompanied with varying proportions of severe cases, whereas most studies lack homogeneity when it came to evaluation of biochemical parameters, pertaining mainly to rapidly evolving guidelines.

As the $\mathrm{PCT}$ release is thought to be inhibited by interferon (INF)- $\gamma$ upsurge, it is expected that the PCT value would remain significantly lower than the optimal cut-off in cases with noncritical or severe infection. ${ }^{3}$ Similarly, in this review, the optimal cut-off was $>0.05 \mathrm{ng} / \mathrm{mL}$ as utilized by studies, i.e., $35 \%(n=18)$ to label severe cases and determine statistical association.

More than $75 \%$ of the studies revealed were conducted in Chinese population. This could be a potential confounding factor 
as the expression of PCT is thought to be dependent upon the genetic framework of the population. ${ }^{6}$ However, a few studies in this review did include the Caucasians and south Asians and similar strong association between PCT and severity was obtained. 7,24,43,59 This multiethnic evaluation further strengthens our proposition of PCT as a prognostic biomarker in COVID-19 cases. However, studies from various populations may be needed to understand the prognostic ability better.

As revealed by Liu et al., PCT levels greater than $0.07 \mathrm{ng} / \mathrm{mL}$ with an AUC of 0.812 and sensitivity and specificity of 73.15 and $84.85 \%$, respectively, for the prediction of morbidity can be considered in routine clinical practice in conjunction with other biochemical markers and clinical picture. ${ }^{32}$ The prediction of cases that can potentially progress to severe stage can aid optimal resource allocation and aggressive treatment plans.

Majority of the studies in this review were inpatient based, making it more feasible to longitudinally record follow-up, which is essential to evaluate prognostic performance of PCT. Furthermore, the population evaluated by the studies in this review was multiethnic ranging from Chinese, Indian, Europeans, North and South Americans, as depicted in Table 1. A few notable limitations include exclusion of non-English publications mostly from China. Existing comorbidities including renal dysfunction, prior bacterial infection, and prophylactic antibiotic initiation that could alter PCT levels were not extensively evaluated. A meta-analysis could not be performed owing to the lack of uniformity of the statistical models adopted by various studies. Moreover, microbiological culture results for coinfecting bacterial and fungal infections were not appraised.

\section{Conclusion}

In spite of the several limitations, PCT seems to appear as a promising prognostic biomarker in COVID-19. Initially elevated PCT levels may be used as a prompt prognosticator of criticalness, deteriorating clinical picture, and even mortality in COVID-19. The biomarker can also serve as a risk stratification tool for intensive resource allocation and aggressive therapeutics in conjunction with clinical details and other biomarkers, in an already overoccupied medical centers globally amidst the crisis. However, to expansively evaluate the prognostic utility of PCT, large-scale cross-sectional multicenter studies are need of time.

\section{HighLights}

- Procalcitonin (PCT) has emerged as a promising prognostic biomarker in COVID-19.

- Various studies have supported the view that PCT levels are below the optimal cut-off in COVID-19 and any considerable increase from baseline reflects the development of a critical state.

- Given these unique characteristics, reliable kinetics, and the potential association of declining levels with resolution of infection, this is a comprehensive systematic review of studies that have evaluated PCT in COVID-19, from across the globe with a total sample size of 15,296 cases.

\section{Authors' Contributions}

Sibtain Ahmed performed the literature search, reviewed articles, data analysis, and write-up of the work in the first draft. Lena Jafri conceived the idea, reviewed articles, and coordinated the writing of the paper and reviewed the final draft. Zahra Hoodbhoy helped with the tables and critically revised the article for the intellectual content. Imran Siddiqui reviewed the articles in case of conflict between Sibtain Ahmed and Lena Jafri and critically revised the article for the intellectual content. All authors have reviewed the final draft and agreed upon.

\section{ACKnOWLedgments}

The authors would like to acknowledge and thanks Mr. Khawaja Mustafa, Librarian, Faculty of Health Sciences, The Aga Khan University, Karachi, Pakistan, for his assistance with literature search.

\section{References}

1. Ahmed S, Jafri L, Majid H, Khan AH, Ghani F, Siddiqui I. Challenges amid COVID-19 times - review of the changing practices in a clinical chemistry laboratory from a developing country. Ann Med Surg (Lond) 2020;55:300-304. DOI: 10.1016/j.amsu.2020.06.004.

2. Jafri L, Ahmed S, Siddiqui I. Impact of COVID-19 on laboratory professionals - A descriptive cross sectional survey at a clinical chemistry laboratory in a developing country. Ann Med Surg (Lond) 2020;57:70-75. DOI: 10.1016/j.amsu.2020.07.022.

3. Ponti G, Maccaferri M, Ruini C, Tomasi A, Ozben T. Biomarkers associated with COVID-19 disease progression. Crit Rev Clin Lab Sci 2020. 1-11. DOI: 10.1080/10408363.2020.1803789.

4. Lin L, Lu L, Cao W, Li T. Hypothesis for potential pathogenesis of SARS-CoV-2 infection-a review of immune changes in patients with viral pneumonia. Emerg Microbes Infect 2020;9(1):727-732. DOI: 10.1080/22221751.2020.1746199.

5. Lima MEDS, Barros LCM, Aragao GF. Could autism spectrum disorders be a risk factor for COVID-19? Med Hypotheses 2020. 144. DOI: 10.1016/j.mehy.2020.109899.

6. Ahmed S, Siddiqui I, Jafri L, Hashmi M, Khan AH, Ghani F. Prospective evaluation of serum procalcitonin in critically ill patients with suspected sepsis - experience from a tertiary care hospital in Pakistan. Ann Med Surg (Lond) 2018;35:180-184. DOI: 10.1016/j. amsu.2018.10.004.

7. Bhandari S, Bhargava A, Sharma S, Keshwani P, Sharma R, Banerjee S. Clinical profile of Covid-19 infected patients admitted in a tertiary care hospital in North India. J Assoc Physicians India 2020;68(5):13-17.

8. Cao H, Ruan L, Liu J, Liao W. The clinical characteristic of eight patients of COVID-19 with positive RT-PCR test after discharge. J Med Virol 2020;92(10):2159-2164. DOI: 10.1002/jmv.26017.

9. Cleland DA, Eranki AP. Procalcitonin. [updated 2020 Sep 3]. StatPearls [Internet]. . Treasure Island (FL): StatPearls Publishing; 2020.

10. Ke C, Wang Y, Zeng X, Yang C, Hu Z. 2019 Novel coronavirus disease (COVID-19) in hemodialysis patients: a report of two cases. Clin Biochem 2020;81:9-12. DOI: 10.1016/j.clinbiochem.2020.04.008.

11. Lippi G, Plebani M. Procalcitonin in patients with severe coronavirus disease 2019 (COVID-19): a meta-analysis. Clin Chim Acta 2020;505:190191. DOI: 10.1016/j.cca.2020.03.004.

12. Li X, Wang L, Yan S, Yang F, Xiang L, Zhu J, et al. Clinical characteristics of 25 death cases with COVID-19: a retrospective review of medical records in a single medical center, Wuhan, China. Int J Infect Dis 2020;94:128-132. DOI: 10.1016/j.ijid.2020.03.053.

13. Moher D, Liberati A, Tetzlaff J, Altman DG, Group P. Preferred reporting items for systematic reviews and meta-analyses: the PRISMA statement. Int J Surg 2010;8(5):336-341. DOI: 10.1016/j. ijsu.2010.02.007.

14. Landis JR, Koch GG. The measurement of observer agreement for categorical data. Biometrics 1977;33(1):159-174. DOI: 10.2307/ 2529310.

15. Hayden JA, Cote P, Bombardier C. Evaluation of the quality of prognosis studies in systematic reviews. Ann Intern Med 2006;144(6):427-437. DOI: 10.7326/0003-4819-144-6-200603210-00010. 
16. Cecconi M, Piovani D, Brunetta E, Aghemo A, Greco M, Ciccarelli $\mathrm{M}$, et al. Early predictors of clinical deterioration in a cohort of 239 patients hospitalized for Covid-19 infection in Lombardy, Italy. J Clin Med 2020;9(5):1548. DOI: 10.3390/jcm9051548.

17. Cen $Y$, Chen $X$, Shen $Y$, Zhang $X H$, Lei $Y, X u$ C, et al. Risk factors for disease progression in patients with mild to moderate coronavirus disease 2019 - a multi-centre observational study. Clin Microbiol Infect 2020;26(9):1242-1247. DOI: 10.1016/j.cmi.2020.05.041.

18. Chen R, Liang W, Jiang M, Guan W, Zhan C, Wang T, et al. Risk factors of fatal outcome in hospitalized subjects with coronavirus disease 2019 from a nationwide analysis in china. Chest 2020;158(1):97-105. DOI: 10.1016/j.chest.2020.04.010.

19. Chen R, Sang L, Jiang M, Yang Z, Jia N, Fu W, et al. Longitudinal hematologic and immunologic variations associated with the progression of COVID-19 patients in China. J Allergy Clin Immunol 2020;146(1):89-100. DOI: 10.1016/j.jaci.2020.05.003.

20. Chen T, Dai Z, Mo P, Li X, Ma Z, Song S, et al. Clinical characteristics and outcomes of older patients with coronavirus disease 2019 (COVID19) in Wuhan, China (2019): a single-centered, retrospective study. J Gerontol A Biol Sci Med Sci 2020;75(9):1788-1795. DOI: 10.1093/ gerona/glaa089.

21. Chen $X$, Yang $Y$, Huang $M$, Liu L, Zhang $X$, Xu J, et al. Differences between COVID-19 and suspected then confirmed SARS-CoV-2negative pneumonia: a retrospective study from a single center. J Med Virol 2020;92(9):1572-1579. DOI: 10.1002/jmv.25810.

22. Duan J, Wang X, Chi J, Chen H, Bai L, Hu Q, et al. Correlation between the variables collected at admission and progression to severe cases during hospitalization among patients with COVID-19 in Chongqing. J Med Virol 2020(11). DOI: 10.1002/jmv.26082.

23. Gavin W, Campbell E, Zaidi SA, Gavin N, Dbeibo L, Beeler C, et al. Clinical characteristics, outcomes and prognosticators in adult patients hospitalized with COVID-19. Am J Infect Control 2020. DOI: 10.1016/j.ajic.2020.07.005S0196-6553(20)30689-1.

24. Gregoriano C, Koch D, Haubitz S, Conen A, Fux CA, Mueller B, et al. Characteristics, predictors and outcomes among 99 patients hospitalised with COVID-19 in a tertiary care centre in Switzerland: an observational analysis. Swiss Med Wkly 2020;150:w20316. DOI: 10.4414/smw.2020.20316.

25. Guo T, Fan Y, Chen M, Wu X, Zhang L, He T, et al. Cardiovascular implications of fatal outcomes of patients with coronavirus disease 2019 (COVID-19). JAMA Cardiol 2020;5(7):811-818. DOI: 10.1001/ jamacardio.2020.1017.

26. Hong Y, Wu X, Qu J, Gao Y, Chen H, Zhang Z. Clinical characteristics of coronavirus disease 2019 and development of a prediction model for prolonged hospital length of stay. Ann Transl Med 2020;8(7):443. DOI: 10.21037/atm.2020.03.147.

27. Hou H, Zhang B, Huang H, Luo Y, Wu S, Tang G, et al. Using IL-2R/ lymphocytes for predicting the clinical progression of patients with COVID-19. Clin Exp Immunol 2020;201(1):76-84. DOI: 10.1111/cei.13450.

28. Hu R, Han C, Pei S, Yin M, Chen X. Procalcitonin levels in COVID-19 patients. Int J Antimicrob Agents 2020(2):106051. DOI: 10.1016/j. ijantimicag.2020.106051.

29. Li H, Xiang X, Ren H, Xu L, Zhao L, Chen X, et al. Serum amyloid A is a biomarker of severe coronavirus disease and poor prognosis. J Infect 2020;80(6):646-655. DOI: 10.1016/j.jinf.2020.03.035.

30. Li R, Tian J, Yang F, Lv L, Yu J, Sun G, et al. Clinical characteristics of 225 patients with COVID-19 in a tertiary hospital near Wuhan, China. J Clin Virol 2020;127:104363. DOI: 10.1016/j.jcv.2020.104363.

31. Lima B, Gibson GT, Vullaganti S, Malhame K, Maybaum S, Hussain ST, et al. COVID-19 in recent heart transplant recipients: clinicopathologic features and early outcomes. Transpl Infect Dis 2020(5):e13382. DOI: 10.1111/tid.13382.

32. Liu $F, L i L, X u M, W u J$, Luo $D, Z$ hu $Y$, et al. Prognostic value of interleukin-6, C-reactive protein, and procalcitonin in patients with COVID-19. J Clin Virol 2020;127:104370. DOI: 10.1016/j.jcv.2020.104370.

33. Liu J, Han P, Wu J, Gong J, Tian D. Prevalence and predictive value of hypocalcemia in severe COVID-19 patients. J Infect Public Health 2020;13(9):1224-1228. DOI: 10.1016/j.jiph.2020.05.029.
34. Luo $X$, Zhou W, Yan $X$, Guo T, Wang B, Xia H, et al. Prognostic value of C-reactive protein in patients with COVID-19. Clin Infect Dis 2020;71(16):2174-2179. DOI: 10.1093/cid/ciaa641.

35. Ma J, Yin J, Qian Y, Wu Y. Clinical characteristics and prognosis in cancer patients with COVID-19: a single center's retrospective study. J Infect 2020;81(2):318-356. DOI: 10.1016/j.jinf.2020.04.006.

36. McRae MP, Simmons GW, Christodoulides NJ, Lu Z, Kang SK, Fenyo D, et al. Clinical decision support tool and rapid point-of-care platform for determining disease severity in patients with COVID-19. Lab Chip 2020;20(12):2075-2085. DOI: 10.1039/DOLC00373E.

37. Ni M, Tian FB, Xiang DD, Yu B. Characteristics of inflammatory factors and lymphocyte subsets in patients with severe COVID-19. J Med Virol 2020;92(11):2600-2606. DOI: 10.1002/jmv.26070.

38. Ortiz-Brizuela E, Villanueva-Reza M, González-Lara MF, TamezTorres KM, Román-Montes CM, Díaz-Mejía BA, et al. Clinical and epidemiological characteristics of patients diagnosed with Covid-19 in a tertiary care center in Mexico city: a prospective cohort study. Rev Invest Clin 2020;72(3):165-177.

39. Pan F, Yang L, Li Y, Liang B, Li L, Ye T, et al. Factors associated with death outcome in patients with severe coronavirus disease-19 (COVID-19): a case-control study. Int J Med Sci 2020;17(9):1281-1292. DOI: 10.7150/ ijms.46614.

40. Price-Haywood EG, Burton J, Fort D, Seoane L. Hospitalization and mortality among black patients and white patients with Covid-19. N Engl J Med 2020;382(26):2534-2543. DOI: 10.1056/NEJMsa2011686.

41. Rastrelli G, Di Stasi V, Inglese F, Beccaria M, Garuti M, Di Costanzo $D$, et al. Low testosterone levels predict clinical adverse outcomes in SARS-CoV-2 pneumonia patients. Andrology 2020. DOI: 10.1111/ andr.12821.

42. Rath D, Petersen-Uribe Á, Avdiu A, Witzel K, Jaeger P, Zdanyte M, et al. Impaired cardiac function is associated with mortality in patients with acute COVID-19 infection. Clin Res Cardiol 2020;109(12):1491-1499. DOI: 10.1007/s00392-020-01683-0.

43. Sattar Y, Connerney M, Ullah W, Rauf H, Mamtani S, Luddington S, et al. Coronavirus disease 2019 with acute respiratory distress syndrome mimicking heart failure exacerbation: time to rethink. Cardiol Res 2020;11(3):196-199. DOI: 10.14740/cr1074.

44. Shao L, Li X, Zhou Y, Yu Y, Liu Y, Liu M, et al. Novel insights into illness progression and risk profiles for mortality in non-survivors of COVID19. Front Med (Lausanne) 2020;7:246. DOI: 10.3389/fmed.2020.00246.

45. Sun D, Li X, Guo D, Wu L, Chen T, Fang Z, et al. CT quantitative analysis and its relationship with clinical features for assessing the severity of patients with COVID-19. Korean J Radiol 2020;21(7):859-868. DOI: 10.3348/kjr.2020.0293.

46. Tian J, Yuan X, Xiao J, Zhong Q, Yang C, Liu B, et al. Clinical characteristics and risk factors associated with COVID-19 disease severity in patients with cancer in Wuhan, China: a multicentre, retrospective, cohort study. Lancet Oncol 2020;21(7):P893-P903. DOI: 10.1016/S1470-2045(20)30309-0.

47. Wan S, Xiang Y, Fang W, Zheng Y, Li B, Hu Y, et al. Clinical features and treatment of COVID-19 patients in northeast Chongqing. J Med Virol 2020;92(7):797-806. DOI: 10.1002/jmv.25783.

48. Wang CZ, Hu SL, Wang L, Li M, Li HT. Early risk factors of the exacerbation of coronavirus disease 2019 pneumonia. J Med Virol 2020(11). DOI: 10.1002/jmv.26071.

49. Wang F, Hou H, Wang T, Luo Y, Tang G, Wu S, et al. Establishing a model for predicting the outcome of COVID-19 based on combination of laboratory tests. Travel Med Infect Dis 2020. 101782. DOI: 10.1016/j. tmaid.2020.101782.

50. Wang F, Yang Y, Dong K, Yan Y, Zhang S, Ren H, et al. Clinical characteristics of 28 patients with diabetes and Covid-19 in Wuhan, China. Endocr Pract 2020;26(6):668-674. DOI: 10.4158/EP-2020-0108.

51. Wu S, Du Z, Shen S, Zhang B, Yang H, Li X, et al. Identification and validation of a novel clinical signature to predict the prognosis in confirmed COVID-19 patients. Clin Infect Dis 2020. ciaa793. DOI: 10.1093/cid/ciaa793.

52. Yan Y, Yang Y, Wang F, Ren H, Zhang S, Shi X, et al. Clinical characteristics and outcomes of patients with severe covid-19 with 
diabetes. BMJ Open Diabetes Res Care 2020(1). DOI: 10.1136/bmjdrc2020-001343.

53. Yang A, Qiu Q, Kong X, Sun $Y$, Chen $T$, Zuo $Y$, et al. Clinical and epidemiological characteristics of COVID-19 patients in Chongqing China. Front Public Health 2020;8:244. DOI: 10.3389/ fpubh.2020.00244.

54. Yang F, Shi S, Zhu J, Shi J, Dai K, Chen X. Clinical characteristics and outcomes of cancer patients with COVID-19. J Med Virol 2020;92(10):2067-2073. DOI: 10.1002/jmv.25972.

55. Yang Q, Xie L, Zhang W, Zhao L, Wu H, Jiang J, et al. Analysis of the clinical characteristics, drug treatments and prognoses of 136 patients with coronavirus disease 2019. J Clin Pharm Ther 2020;45(4):609-616. DOI: 10.1111/jcpt.13170.

56. Ye W, Chen G, Li X, Lan X, Ji C, Hou M, et al. Dynamic changes of $\mathrm{D}$-dimer and neutrophil-lymphocyte count ratio as prognostic biomarkers in COVID-19. Respir Res 2020;21(1):169. DOI: 10.1186/ s12931-020-01428-7.

57. Yu C, Lei Q, Li W, Wang X, Liu W, Fan X, et al. Clinical characteristics, associated factors, and predicting COVID-19 mortality risk: a retrospective study in Wuhan, China. Am J Prev Med 2020(2). DOI: 10.1016/j.amepre.2020.05.002.

58. Yuan X, Huang W, Ye B, Chen C, Huang R, Wu F, et al. Changes of hematological and immunological parameters in COVID-19 patients. Int J Hematol 2020. 1-7.
59. Zaninotto M, Mion MM, Cosma C, Rinaldi D, Plebani M. Presepsin in risk stratification of SARS-CoV-2 patients. Clin Chim Acta 2020;507:161-163. DOI: 10.1016/j.cca.2020.04.020.

60. Zeng Z, Ma Y, Zeng $H$, Huang $P$, Liu W, Jiang $M$, et al. Simple nomogram based on initial laboratory data for predicting the probability of ICU transfer of COVID-19 patients: multicenter retrospective study. J Med Virol 2020.

61. Zhang B, Zhang J, Chen $\mathrm{H}$, Chen L, Chen Q, Li M, et al. Novel coronavirus disease 2019 (COVID-19): relationship between chest CT scores and laboratory parameters. Eur J Nucl Med Mol Imaging 2020;47(9):2083-2089. DOI: 10.1007/s00259-020-04854-3.

62. Zhang G, Hu C, Luo L, Fang F, Chen Y, Li J, et al. Clinical features and short-term outcomes of 221 patients with COVID-19 in Wuhan, China. J Clin Virol 2020;127:104364. DOI: 10.1016/j.jcv.2020.104364.

63. Zhang J, Cao Y, Tan G, Dong X, Wang B, Lin J, et al. Clinical, radiological and laboratory characteristics and risk factors for severity and mortality of 289 hospitalized COVID-19 patients. Allergy 2020. DOI: 10.1111/all.14496.

64. Zhang J, Dong $X$, Cao $Y$, Yuan $Y$, Yang $Y$, Yan $Y$, et al. Clinical characteristics of 140 patients infected with SARS-CoV-2 in Wuhan, China. Allergy 2020;75(7):1730-1741. DOI: 10.1111/all.14238.

65. Assicot M, Gendrel D, Carsin H, Raymond J, Guilbaud J, Bohuon C. High serum procalcitonin concentrations in patients with sepsis and infection. Lancet 1993;341(8844):515-518. DOI: 10.1016/01406736(93)90277-N. 